\title{
A mesothelioma presenting with multicentric reticulohistiocytosis
}

\author{
D. Honeybourne and J.K. Kellett \\ Department of Chest Medicine, Wythenshawe Hospital, Manchester and the Skin Hospital, Manchester, UK.
}

\begin{abstract}
Summary: Multicentric reticulohistiocytosis is a rare disorder of the skin and joints, with characteristic histological features. An increasing number of reported cases have been associated with internal malignancy. We report a case of multicentric reticulohistiocytosis in an elderly man with mesothelioma.
\end{abstract}

\section{Introduction}

Multicentric reticulohistiocytosis (MR) is a rare disorder mainly affecting the skin and joints. There are usually papulonodular skin lesions which mainly affect the upper half of the body especially the extensor surfaces of the arms and hands, and also the scalp and ears. The arthritis may precede the skin lesions in many cases (Barrow \& Holubar, 1969) and tends eventually to progress to joint destruction. There have been several reports of a link between MR and internal malignancies.

\section{Case report}

A man presented at the age of 72 years with an eruption of firm subcutaneous nodules on the dorsa of the fingers (Figure 1). Within a few weeks this extended to involve the hands, temples, ears, neck, shoulders and upper chest. In places the nodules coalesced to form rough, indurated, erythematous plaques. On his initial presentation the patient admitted to some stiffness of the fingers. Subsequently he experienced increasing arthralgia of the interphalangeal and metacarpophalangeal joints and of the knees and wrists. He had been a non-smoker for the previous 40 years. He had worked as a joiner for a firm fitting acoustic insulation and had been exposed to both chrysotile and crocidolite asbestos, the latter some 30 years previously.

Examination revealed reduced breath sounds and percussion note at his left lung base and skin lesions typical of MR. A biopsy of the dorsum of one hand

D. Honeybourne, M.D., M.R.C.P.; and J.K. Kellett, M.B., M.R.C.P.

Correspondence: D. Honeybourne, Department of Chest Medicine, Wythenshawe Hospital, Manchester M23 9LT. Accepted: 2 November 1983 revealed a highly vascular tumour of the mid dermis, composed of histiocytes and pale-staining giant cells, considered typical of reticulohistiocytoma (Figure 2). A synovial biopsy from the knee joint showed similar features. Chest X-ray revealed an homogenous opacity situated peripherally and laterally in the left lower and mid zone, and bilateral pleural calcification. Full blood count and erythrocyte sedimentation rate were normal. A fibreoptic bronchoscopy showed no abnormality but a transthoracic needle biopsy confirmed the diagnosis of mesothelioma. The patient has been followed up for ten months, and has developed increasing left sided chest pain with a concomitant increase in the size of the mass on his chest X-ray. There has been some improvement in his arthralgia following the commencement of oral prednisolone $10 \mathrm{mg}$ daily.

\section{Discussion}

MR has been recognized at least since 1937 (Weber \& Freudenthal, 1937) and the term 'multicentric reticulohistiocytosis of the skin and synovia' was later proposed and a review of the disease including its early history was reported (Goltz \& Laymon, 1954). More recently 33 patients have been described in detail (Barrow \& Holubar, 1969). The clinical syndrome includes nodular lesions of the skin and subcutaneous tissues, mucosa, and synovia. Histologically all these lesions are characterized by the presence of palestaining histiocytic multinucleate giant cells. Females may be more commonly affected than males and the onset of the disease is usually in middle age (Barrow \& Holubar, 1969; Chevrant-Breton, 1977). Initially there is rapid progression of both skin and joint lesions, the latter leading to an incapacitating and deforming arthritis. If the course of the disease is not 


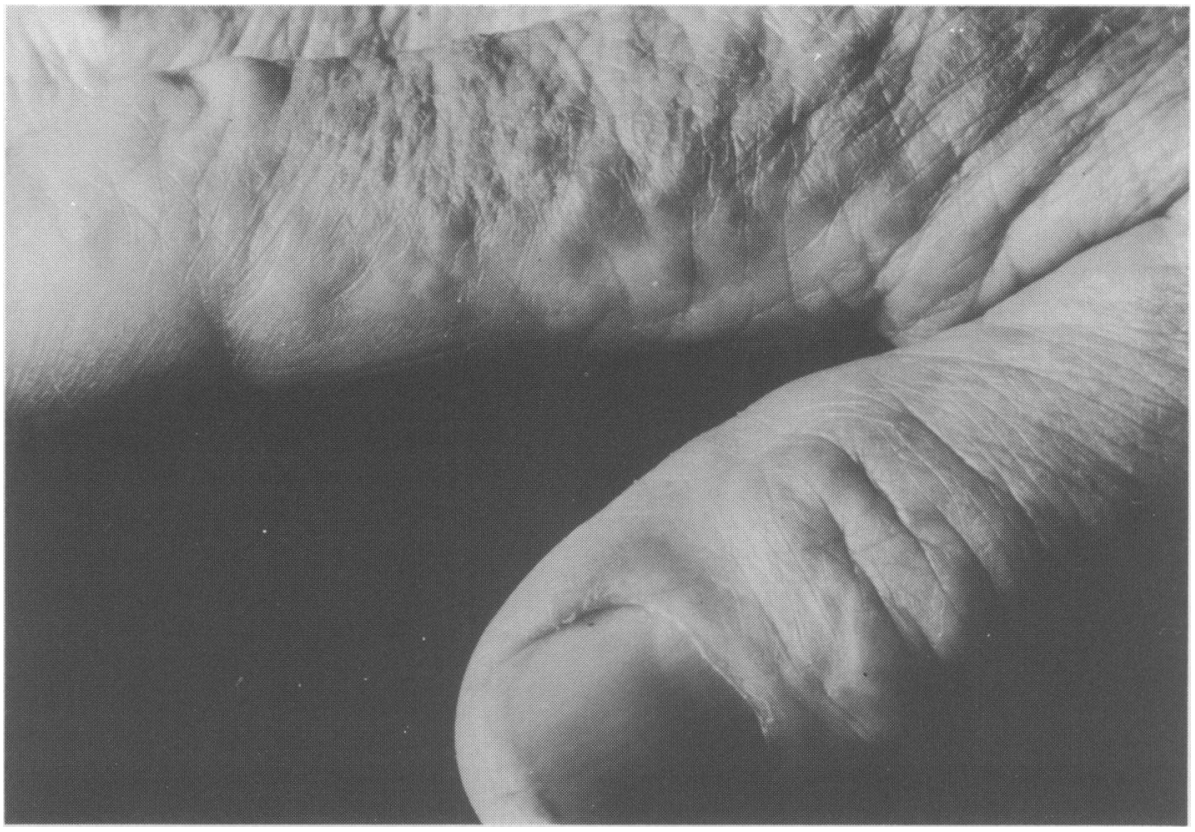

Figure 1 Subcutaneous nodules of multicentric reticulohistiocytosis in the fingers.

interrupted by malignancy, the condition appears to undergo spontaneous remission within a few years in most cases.

The presentation of MR in our patient is atypical in two respects: the relatively late age of onset, and the fact that the arthropathy was always mild. Nevertheless the characteristic histological findings in the synovial biopsy confirm the diagnosis. An association with cardiopulmonary disorders, especially pleural effusions, pulmonary infiltrates and pericarditis has been reported (Fast, 1976).

Concomitant malignancy was found in $5(15 \%)$ of 33 patients with MR (Barrow \& Holubar, 1969). Since then, evidence that the disease is positively associated with malignancy has grown and a more recent review described $18(24 \%)$ out of 74 patients with MR as having associated neoplasia (Catterall,
1980). These included one case of bronchial carcin oma, the others including carcinoma of stomach breast, cervix, colon and ovary. This is the first reported association of MR with a mesothelioma. It is not clear from existing reports whether removal or cure of an underlying malignancy may lead to remission of MR, since most patients with malignancy have succumbed. In at least one patient MR remitted, after removal of a cervical tumour, only to recur a few months later (Catterall, 1980). Prednisolone appears to produce symptomatic improvement of the arthropathy but usually not to affect the overall course. If the skin lesions and arthritis become severe then a trial of cyclophosphamide is justified as a small proportion of cases seem to respond favourably (Hanauer, 1972). 


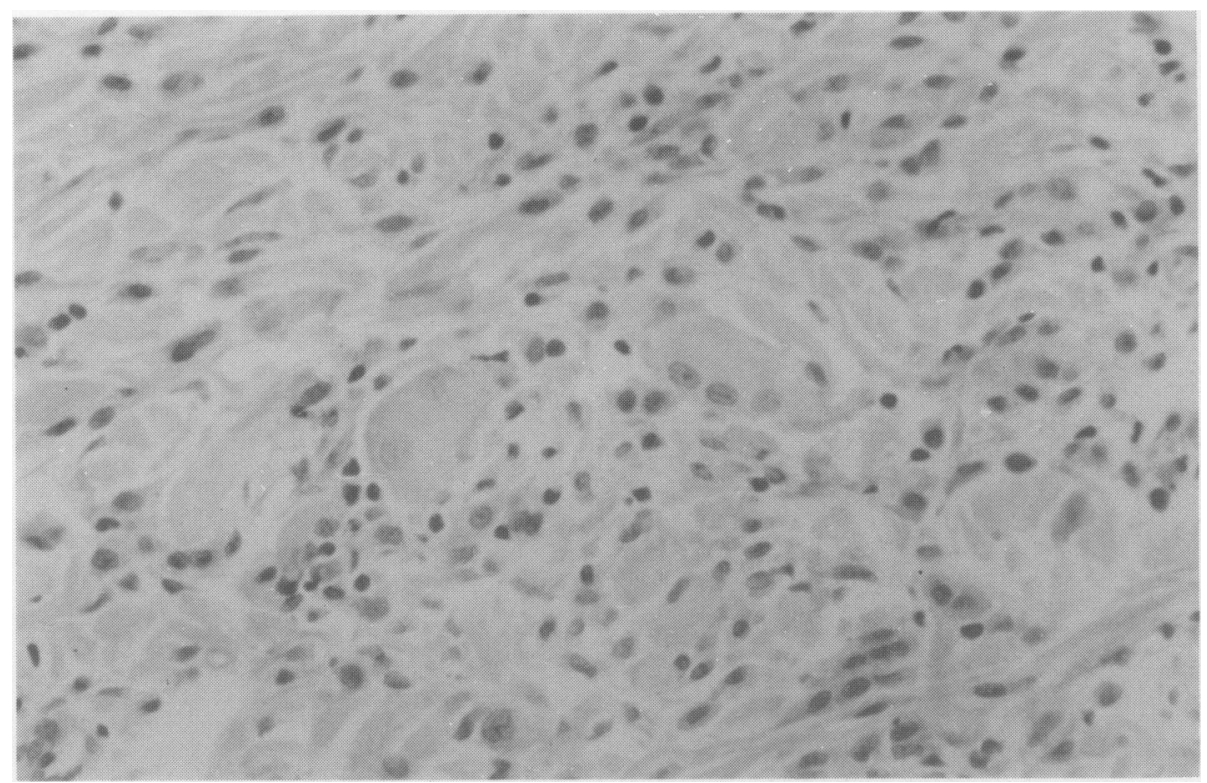

Figure 2 Skin biopsy showing large pale staining giant cells in the mid-dermis $(\times 800)$.

\section{References}

BARROW, M.V. \& HOLUBAR, K. (1969). Multicentric reticulohistiocytosis; a review of thirty-three patients. Medicine, 48, 287.

CATTERALI , M.D. (1980). Multicentric reticulohistiocytosis, a review of eight cases. Clinical and Experimental Dermatology, 5, 267.

CHEVRANT-BRETON, J. (1977). La reticulo-histiocytose multicentrique, revue de la litterature recente (depuis 1969) Annals of Dermatology and Venereology, 104, 745.

FAST, A. (1976). Cardiopulmonary complications in multicentric reticulo-histiocytosis. Archives of Dermatology, $112,1139$.

GOLTZ, R.W. \& LAYMON, C.W. (1954). Multicentric reticulohistiocytosis of the skin and synovia. Archives of Dermatology and Syphilis, 69, 717.

HANAUER, L.B. (1972). Reticulohistiocytosis. Remission after cyclophosphamide therapy. Arthritis and Rheumatism, 15, 636.

WEBER, F.P. \& FREUDENTHAL, W. (1937). Nodular nondiabetic cutaneous xanthomatosis with hypercholesterolaemia and atypical histological features. Proceedings of the Royal Society of Medicine, 30, 522. 